\title{
Identidad de Género en estudios de Ingeniería en el ámbito industrial: una mirada desde la ETSII-UPV hacia las jóvenes generaciones
}

López-Jiménez, P. Amparo; Alemany, MME; González-Cruz, M. C.; García-Serra, J Escuela Técnica Superior de Ingenieros Industriales. Universitat Politècnica de València.

\begin{abstract}
This document analyzes the presence of women in industrial studies at the Technical School of Industrial Engineers (ETSII) of the Universitat Politècnica de València. By reviewing the large numbers of women in the STEM degrees and presenting in more detail the particular indicators in the ETSII, it is intended to emphasize the importance of the presence of women in this type of university studies with an identity number of presence $30 \%$ in such studies. This must be of paramount importance as part of the implementation of the United Nation's fifth sustainable development objective: Achieve gender equality and empowering all women and girls.
\end{abstract}

Keywords: Gender Identity, STEM Degrees, Women and Engineering

\begin{abstract}
Resumen
El presente documento analiza la presencia femenina en los estudios del ámbito industrial en la Escuela Técnica Superior de Ingenieros Industriales (ETSII) de la Universitat Politècnica de València. Haciendo un repaso de los grandes números de presencia de mujeres en las titulaciones STEM, y presentando con más detalle los indicadores particulares en la ETSII, se desea hacer hincapié sobre la importancia de la presencia femenina en este tipo estudios universitarios con un número identitario del 30\% de mujeres en dichas titulaciones. Este debe ser un interés esencial de la aplicación del quinto objetivo de desarrollo sostenible de Nacionas Unidas: Lograr una igualdad entre los géneros y empoderar a todas las mujeres y las ninas.
\end{abstract}

Palabras clave: Identidad de género, Titulaciones STEM, Mujer e Ingeniería

\section{Introducción}

La educación superior no es ajena a la evolución de la sociedad, y de la misma forma que esta sociedad en sus inicios marca una diferencia entre clases sociales y género, la Universidad la refleja desde sus inicios. Mucho ha sido el terreno avanzado en la consecución de una igualdad social en las Universidades españolas y más recientemente hacia la presencia de las mujeres en todos los estamentos de las mismas (Elizondo Lopetegui et al. 2010).

Sin embargo, la presencia femenina en la Universidad no solo ha sido consecuencia de la evolución natural de la sociedad hacia la igualdad, sino que se ha promocionado de forma institucional. Este aspecto ha sido analizado en diversos informes desde hace décadas 
(Fernández Villanueva, 1989) y trae como consecuencia acciones institucionales en aras de la igualdad. En 1999 se promocionó desde la Union Europea la iniciativa Helsinki Women and Science para analizar el papel de las mujeres en la ciencia y la investigación, y promover medidas en el marco de la UE hacia la integración (Bebbington, (2002). Desde este momento, muchas han sido las iniciativas para conseguir una mayor presencia femenina en los estamentos universitarios desde todos los ámbitos administrativos. En el marco de estas iniciativas locales o nacionales, se encuentra la determinación de los Objetivos de Desarrollo Sostenible (ODS), presentados en septiembre de 2015 en la histórica Cumbre del desarrollo sostenible en que se aprobó la Agenda 2030 (Naciones Unidas, 2015).

Tabla 1. Objetivos de Desarrollo Sostenible (Naciones Unidas, 2015).

\begin{tabular}{|c|}
\hline $\begin{array}{c}\text { 2. Poner fin al hambre, lograr la seguridad alimentaria y la mejora de la nutrición y } \\
\text { promover la agricultura sostenible }\end{array}$ \\
\hline 3. Garantizar una vida sana y promover el bienestar para todos en todas las edades \\
\hline $\begin{array}{l}\text { 4. Garantizar una educación inclusiva, equitativa y de calidad y promover oportunidades de } \\
\text { aprendizaje durante toda la vida para todos }\end{array}$ \\
\hline 5. Lograr la igualdad entre los géneros y empoderar a todas las mujeres y las niñas \\
\hline 6. Garantizar la disponibilidad de agua y su gestión sostenible y el saneamiento para todos \\
\hline 7. Garantizar el acceso a una energía asequible, segura, sostenible y moderna para todos \\
\hline $\begin{array}{l}\text { 8. Promover el crecimiento económico sostenido, inclusivo y sostenible, el empleo pleno y } \\
\text { productivo y el trabajo decente para todos }\end{array}$ \\
\hline $\begin{array}{l}\text { 9. Construir infraestructuras resilientes, promover la industrialización inclusiva y sostenible } \\
\text { y fomentar la innovación }\end{array}$ \\
\hline 10. Reducir la desigualdad en y entre los países \\
\hline $\begin{array}{l}\text { 11. Lograr que las ciudades y los asentamientos humanos sean inclusivos, seguros, } \\
\text { resilientes y sostenibles }\end{array}$ \\
\hline 12. Garantizar modalidades de consumo y producción sostenibles \\
\hline 13. Adoptar medidas urgentes para combatir el cambio climático y sus efectos \\
\hline $\begin{array}{l}\text { 14. Conservar y utilizar en forma sostenible los océanos, los mares y los recursos marinos } \\
\text { para el desarrollo sostenible }\end{array}$ \\
\hline $\begin{array}{l}\text { 15. Proteger, restablecer y promover el uso sostenible de los ecosistemas terrestres, gestionar } \\
\text { los bosques de forma sostenible, luchar contra la desertificación, detener e invertir la } \\
\text { degradación de las tierras y poner freno a la pérdida de la diversidad biológica }\end{array}$ \\
\hline $\begin{array}{l}\text { 16. Promover sociedades pacíficas e inclusivas para el desarrollo sostenible, facilitar el } \\
\text { acceso a la justicia para todos y crear instituciones eficaces, responsables e inclusivas a } \\
\text { todos los niveles }\end{array}$ \\
\hline $\begin{array}{l}\text { 17. Fortalecer los medios de ejecución y revitalizar la Alianza Mu } \\
\text { Sostenible }\end{array}$ \\
\hline
\end{tabular}

Los ODS constituyen un desiderátum de 17 metas de aplicación universal con el objetivo de focalizar los esfuerzos de los países para lograr un mundo sostenible en el año 2030. El total de estos objetivos está descrito en la Tabla 1. 
En el marco de los objetivos descritos se encuentra el quinto de ellos, destinado a lograr la igualdad de género y empoderar a todas las mujeres y niñas. No cabe duda de que la educación superior y la presencia más notable de la mujer en los estamentos de las Escuelas de Ingeniería es una forma de intentar cumplir dicho objetivo en el seno de una sociedad que requiere de la presencia de la mujer en cualquiera de sus espacios.

\section{¿Cuál es la relación entre mujeres e ingeniería?}

La presencia de mujeres en el marco de los estudios de Ciencias, Tecnología, Ingeniería y Matemáticas (STEM) es menor que la de hombres.

Los grandes números indican que, en promedio, la presencia de mujeres en titulaciones superiores STEM es de aproximadamente el 30\%. En datos de alumnos egresados, la proporción del numero identitario del 30,5\% de mujeres está igualmente presente en los estudios de ingeniería (Ministerio de Ciencia, Innovación y Universidades, 2017). La razón de ello hay que buscarla en la etapa de la niñez de estas mujeres, antes de que tengan que tomar decisiones sobre sus inclinaciones laborales. En muchas ocasiones, son los estereotipos de género presentados a las niñas en su infancia y adolescencia los que hacen que se decidan por otro tipo de titulaciones. Sin embargo, estas titulaciones tienen un gran futuro y empleabilidad, y la vinculación de las mujeres con el éxito de este tipo de enseñanzas debe ser un compromiso de esta sociedad con una mitad de la población que puede enriquecer cualquiera de sus ámbitos.

Las cifras presentadas anteriormente constantan que ese tercio de presencia femenina en carreras STEM es un hecho continuado y prácticamente cierto a muchas escalas. Esta proporción se traslada, por tanto, a las empresas de base tecnológica, de forma que estas son mayoritariamente masculinas puesto que las mujeres que pueden acceder a esa fuerza de trabajo también son un tercio aproximadamente de las personas que pueden hacerlo. (Rodríguez, L. 2017)

\section{Los inicios de la presencia de mujeres en la Ingeniería Industrial española}

La profesión de Ingeniero Industrial viene regulada desde 1850. El Real Decreto de 4 de septiembre de 1850 "sobre la creación de la carrera de Ingenieros Industriales. Programa de la enseñanza. Cuadro de Profesores", presentado por el Ministro de Comercio, Instrucción y Obras Públicas, D. Manuel de Seijas Lozano, da realidad a una profesión que venia impartiéndose en diversas instituciones desde hacía algunos años, tales como el Seminario Patriótico de Vergara, desde 1774. (Sebastián Pérez, 2015).

Sin embargo la presencia femenina en las Escuelas de Ingenieros Industriales en España no ocurre hasta mucho después. La primera Ingeniera Industrial se titula en las aulas de la Escuela de Ingenieros Industriales de Madrid en 1929. Este año, la alumna Pilar Careaga y Basabe se convierte en la primera Ingeniera Industrial, a la temprana edad de 21 años. Mujer con una gran vocación de tecnóloga, desde los 13 años manifestó a su entorno familiar su gran interés y capacidad para las matemáticas, la física y el álgebra, siendo su gran pasión las 
locomotoras, hecho muy poco frecuente en 1921, aunque con más frecuencia hoy en día. (Escuela de Ingenieros Industriales de Madrid, 2011).

Desde 1929 el panorama universitario y la indentidad de género han evolucionado. Sin embargo, la presencia de mujeres en carreras puramente tecnológicas dista de ser igual a la de los hombres. Pero como ya se observa en el ejemplo de esta pionera de la Ingeneiria Industrial en España, la clave está en esa edad temprana en que las personas deciden su futura vocación.

La Escuela de Ingenieros Industriales (ETSII) de la Universitat Politèncnica de València nació en 1968. Sin embargo, no es hasta 1983 cuando en su décima promoción se titula la primera mujer. En los años siguientes hasta la década de los 90, la presencia de mujeres es mínima entre los titulados.

Sin embargo, a partir de 1993 la presencia femenina entre las solicitudes de ingreso a la ETSII es más significativa. La presente contribución describe estos ingresos en los años más recientes.

\section{Objetivos}

La presente comunicación describe la tasa de participación femenina entre el alumnado de ingreso en la ETSII de la Universitat Politècnica de València y presenta algunas de las iniciativas llevadas a cabo para atraer a niñas y jóvenes estudiantes hacia las titulaciones impartidas en la citada Escuela.

\section{Desarrollo de la Innovación}

Las tasas de participación en las diferentes titulaciones ofertadas en la ETSII dan como consecuencia una presencia media de un 30,38\% de mujeres entre las solicitudes de ingreso teniendo en cuenta los registros de matrícula desde 1993 hasta la actualidad. Esto es, como se ve, completamente coherente con el informe de 2017 descrito por el Ministerio de Ciencia, Innovación y Universidades que presenta para el curso 2016-2017 un porcentaje del 30,5\% de mujeres en estudios universitarios de ingeniería, industria y construcción.

Sin embargo la presencia femenina por titulaciones es dispar. Mientras que la presencia media entre los ingresos desde 1993 y 2009 de mujeres en la titulación de Ingeniero Industrial es del 24,5\%, en la titulación de Ingeniero Quimico es del 55\% en el mismo período.

A partir de 2011 se ponen en marcha los diferentes grados bajo el marco del proceso de construcción del Espacio Europeo de Educación Superior, y desde ese momento la solicitud de mujeres para el ingreso en la ETSII es igualmente dispar. Como promedio, desde 2011 a 2018, un 27,65\% de mujeres ingresaron en Grado en Ingeniería de la Energía; un 58,62\% en Grado en Ingeniería Biomédica; un $34,7 \%$ lo hicieron en el Grado en Ingeniería en Organización Industrial; un 43,5\% en el Grado en Ingeniería Química y un 22,87\% en el Grado en Ingeniería en Tecnologías Industriales, que es la titulación equivalente, junto con el Máster Ingeniero Industrial, al Ingeniero Industrial anterior al EEES. Cabe destacar que en el conjunto de los grados, en estos nuevos planes de estudios la proporción de mujeres en 
datos de ingreso incluso descendió con respecto a las titulaciones similares en planes previos. El paso de los años no ha significado un incremento en la captación de talento femenino.

Se podría indicar así que la identidad de género en la ETSII es cercana al 30\%, sin incrementarse de forma notable en los últimos años, por lo que la captación de talento debe ser realizada hacia las niñas y estudiantes que tienen que decantarse por una formación tecnológica en fases previas de su formación: es absolutamente imprescindible mirar hacia las niñas y jóvenes para que se planteen las titulaciones de ingenera química, de la energía, biomédica, de organización o en las tecnologías industriales como una posibilidad real y atractiva. El desarrollo de la innovación proviene de un análisis de la evolución y situación actual de la identidad de género en la ETSII-UPV, aspecto del que no se tiene constancia haya sido analizado con anterioridad. En realidad éste es el primer paso (hacer un diagnóstico de la situación actual) para emprender las acciones correspondientes en adelante.

\section{Resultados}

Muchas son las acciones a considerar en aras de captar talento en edades más tempranas. A modo de ejemplo, la iniciativa PRAKTIKUM se inció en 2010, entre otros centros, en la ETSII (Universitat Politècnica de València, 2019). Esta iniciativa tiene por objeto favorecer la presencia en el Centro de estudiantes procedentes de primero de bachiller o ciclos formativos. El alumnado joven accede a talleres, actividades y trabaja con los profesores de la UPV. En este sentido los programas han versado sobre aspectos de las diversas titulaciones que se ofertan en la ETSII y han contado con una notable presencia femenina, con una evolución positiva en los últimos años, a pesar del pequeño número de alumnos que accede en cada programa.

Para edades más temprana, y desde 2015, en la UPV existen proyectos similares. Concretamente, Experimenta-Escola d'Estiu UPV, incluye actividades adaptadas a la edad de los participantes. De este modo, incluso los más pequeños -de 3 a 6 años- están haciendo ya sus primeros pinitos tecnológicos en actividades adaptadas a su edad, tales como dar vida a pequeñas abejas (bee-bots) robotizadas. Dentro del mismo ámbito, niños y niñas de entre 10 y 12 años programan sus propios robots para realizar diferentes retos de la forma más rápida. Existen multitud de iniciativas dedicadas a la captación de niñas y jóvenes a nivel institucional, comenzando por los programas llevados a cabo desde la UPV. El programa up!Steam (Universitat Politècnica de València, 2018) tiene por objeto despertar en incentivar las vocaciones de manera igualitaria en niños y niñas hacia las titulaciones científicas, tecnológicas y artísitcas.

Otro ejemplo a nivel nacional es la iniciativa «Quiero ser Ingeniera» en la que participan ciertas Unviersidades (Universidad Politécnica de Cartagena, 2019). Es este un proyecto "para fomentar las vocaciones científicas y tecnológicas, especialmente entre las chicas de Educación Secundaria Obligatoria (ESO)".

En la misma dirección, en 2016 se crea el conjunto de Acciones Mujer e Ingeniería desde la Real Academia de Ingeniería con "el objetivo de motivar e interesar a niñas y adolescentes, fomentando las vocaciones en estudios STEM de forma que se favorezca la incorporación de más mujeres a las distintas especialidades profesionales relacionadas con la ingeniería y el 
desarrollo de su recorrido profesional hasta alcanzar puestos de responsabilidad." (Real Academia de Ingeniería, 2016). Yendo más allá, es necesario establecer mecanismos que midan el impacto de las diversas iniciativas que se llevan a cabo en las universidades o en otros niveles para medir su eficacia. Se podría así parametrizar cómo estos han ido impactando a lo largo de las diferentes iniciativas para detectar aquellas acciones que sean más eficaces.

\section{Conclusiones}

La presencia de mujeres en las titulaciones del ámbito de la ingeniería industrial en la ETSII de la UPV se encuentra entre un 25 y un 30\% como media, con la exepción del Grado en Ingeniería Química y el Grado en Ingeniería Biomédica en los que la presencia de mujeres y hombres es casi igualitaria. Se espera que en años venideros la proporción de mujeres en todos los grados aumente. Debe dirigirse una mirada optimista hacia las jóvenes generaciones que tienen que seleccionar estos grados, impulsando desde los diferentes estamentos sociales la importancia que tiene que niñas y adolescentes se den cuenta que se encuentran perfectamente capacitadas y dotadas para seguir estas titulaciones, haciéndolas una alternativa atractiva a su vocación. Existen en esta dirección multitud de iniciativas, cuyos resultados se verán en los próximos años, puesto que la escasez de mujeres en el ámbito de la ingeniería y la tecnología conlleva un desaprovechamiento implícito de su potencial creativo. La presencia de mujeres en las titulaciones del ámbito industrial en el marco de los estudios STEM, es coherente con el desarrollo normal de una sociedad que tiene como objetivo de desarrollo sostenible lograr la igualdad entre los géneros y empoderar a todas las mujeres y las niñas.

\section{Referencias}

BEBBINGTON, D. (2002), "Women in Science, Engineering and Technology: A Review of The Issues. " Higher Education Quarterly, 56: 360-375. doi:10.1111/1468-2273.00225

ELIZONDO LOPETEGUI, A.; NOVO ARBONA, A.; SILVESTRE CABRERA, M. (2010). "Igualdad de hombres y mujeres en las Universidades Españolas." Instituto de la Mujer. Madrid.

ESCUELA TÉCNICA SUPERIOR DE INGENIEROS INDUSTRIALES DE MADRID. (2011) La Primera Mujer Ingeniera se tituló en la Escuela. $<$ https://www.escuelaindustrialesupm.com/ingenieroindustrial/la-primera-mujer-ingeniera-se-titulo-en-la-escuela/> [Consulta: marzo de 2019]

FERNÁNDEZ VILLANUEVA, C.(1989). "La mujer en la universidad española: docencia, investigación y poder”. Revista de Educación, no 290 (1989), pp. 161-171.

MINISTERIO DE CIENCIA, INNOVACIÓN Y UNIVERSIDADES (2017) Estadísticas Universitarias. $\quad$ http: //www.educacionyfp.gob.es/servicios-al-ciudadanomecd/estadisticas/educacion/universitaria/estadisticas/alumnado.html> [Consulta: marzo de 2019]

NACIONES UNIDAS (2015). "Transformar nuestro mundo: la Agenda 2030 para el Desarrollo Sostenible. Resolución aprobada por la Asamblea General el 25 deseptiembre de 2015. A/RES/70/1, 21 de octubre." 
REAL ACADEMIA DE INGENIERÍA, 2016. Acciones Mujer e Ingeniería. Consultado en Internet en marzo de 2019 en: http://www.raing.es/es/content/acciones-mujer-e-ingenier

RODRÍGUEZ, LUZ (2017). ¿Por qué las mujeres no estudian Ingeniería?.

$<$ https://www.infolibre.es/noticias/luces_rojas/2017/10/25/por_que_las_mujeres_no_estudian_ ingenieria_71057_1121.html> [Consulta: marzo de 2019]

SEBASTIÁN PÉREZ, M.A. 2015. "Historia de las enseñanzas de la Ingeniería Industrial en España". $<$ https://riuma.uma.es/xmlui/bitstream/handle/10630/10644/PresentacionMASP UMA.pdf?sequence $=2>$ [Consulta: marzo de 2019]

UNESCO. 2017 (Bokova, I.G.). writer of foreword. "Cracking the code: girls' and women's education in science, technology, engineering and mathematics (STEM)".

UNIVERSITAT POLITÈCNICA DE VALÈNCIA. 2019. PRAKTIKUM UPV.: $<$ http://www.upv.es/contenidos/PRAKTIKUM/> [Consulta: marzo de 2019]

UNIVERSITAT POLITÈCNICA DE VALÈNCIA, 2018. Up!Steam.: < https://www.upv.es/noticiasupv/noticia-10607-up-steam-es.html> [Consulta: marzo de 2019]

UNIVERSIDAD POLITÉCNICA DE CARTAGENA, 2019. Quiero ser Ingeniera. $<\underline{\text { https://quieroseringeniera.upct.es/> [Consulta: marzo de 2019] }}$ 